\title{
Tungsten sputtering and accumulation of implanted carbon and deuterium by simultaneous bombardment with $D$ and $C$ ions
}

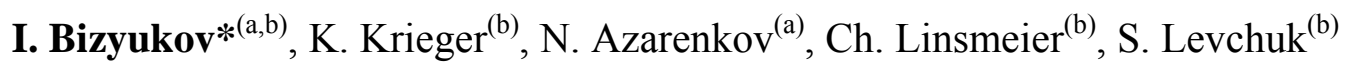 \\ ${ }^{(a)}$ Kharkiv National University, Faculty of Physics and Technologies, 31 Kurchatov Ave., \\ Kharkiv 61108, Ukraine \\ ${ }^{(b)}$ Max-Planck-Institut für Plasmaphysik, EURATOM Association, Boltzmannstr. 2, 85748 Garching, \\ Germany
}

\begin{abstract}
Sputtering of tungsten by simultaneous incidence of gaseous and non-volatile ions is an important field of research for nuclear fusion with magnetically confined plasmas. In order to investigate the underlying processes in detail, W layers deposited on graphite and $\mathrm{Si}$ substrates have been irradiated simultaneously with beams of $12 \mathrm{keV} C_{2}^{-}$and $9 \mathrm{keV} D_{3}^{+}$ions. The dynamics of $\mathrm{W}$ sputtering as well as the accumulation of implanted $\mathrm{C}$ and $\mathrm{D}$ was studied in-situ by ion beam analysis (IBA) using $2.5 \mathrm{MeV}{ }^{3} \mathrm{He}^{+}$ions. In this work, particularly the sputter yield of $\mathrm{W}$ and the implantation of $\mathrm{C}$ and $\mathrm{D}$ as a function of the $\mathrm{C}$ fraction in the incident flux is discussed. Comparison of experimental data to TRIDYN simulations reveal a strong contribution of surface roughness to $\mathrm{W}$ sputtering and $\mathrm{C}$ implantation. In comparison to the influence of roughness, the contribution of chemical effects appears negligible.
\end{abstract}


PACS: $79.20 \mathrm{Rf}$

JNM keywords: Tungsten, Deuterium, Carbon, Ion Irradiation.

PSI-17 keywords: Tungsten, Sputtering, Deuterium inventory, Carbon impurities, Surface analysis.

*Corresponding author address: Boltzmannstraße2, D-85748 Garching, Germany.

*Corresponding author E-mail: ivan.bizyukov@ipp.mpg.de

Presenting author: Bizyukov Ivan

Presenting author e-mail: ivan.bizyukov@ipp.mpg.de 


\section{Introduction}

All major design studies of future fusion research and reactor devices employ several different plasma facing materials depending on the vessel location [1]. For regions subject to high particle fluxes such as divertor and baffle structures tungsten is envisaged as plasmafacing material as reported e.g. in [2, 3, 4]. Therefore, bombardment of tungsten by simultaneous incidence of carbon and deuterium ions is an important field of research for nuclear fusion.

Results from the ASDEX-Upgrade tungsten-divertor experiment show that the erosion of tungsten is dominated by impact of impurity ions where $\mathrm{C}, \mathrm{W}$ and $\mathrm{O}$ are the most common species [5]. With simultaneous bombardment by ions of D and C projectiles the sputtering processes and target modification will depend on the ratio of the incident species and will for example lead to a competition between $\mathrm{W}$ erosion and growth of a protective layer containing carbon and deuterium [6]. In this respect, the balance point between the two behaviours will be of particular importance, since it separates two qualitatively different processes. The physics model for the description of the surface erosion becomes more complicate because the properties of the material mix ( $\mathrm{W}$ and $\mathrm{C}$ with a minor fraction of $\mathrm{D}$ ) will be generally different from those of the original target material.

Laboratory weight-loss experiments on simultaneous bombardment of high- $Z$ materials with hydrogen isotopes and carbon projectiles have been reported previously [7, 8]. The experimental results, particularly regarding total erosion yields, cannot be explained by the linear superposition of processes resulting from mutually independent irradiation of tungsten with carbon and hydrogen [7]. The understanding of the processes involved was further developed and reported in [8]. One observes that pure carbon bombardment leads to continuous tungsten erosion only at elevated temperatures of $1073 \mathrm{~K}$, where diffusion causes 
continuous depletion of carbon from the surface. This effect starts already at much lower temperature in presence of incident hydrogen ions. However, direct experiments with variable $\mathrm{C}$ fraction in the incident flux are not possible with installations producing single ion beam.

A new Dual Beam Experiment (DBE) has been designed and implemented at IPP Garching, which allows a much wider parameter range and species selection, than accessible in previous experiments [9]. As an improvement over existing comparable devices [10], it allows for the first time in-situ ion beam analysis of irradiated samples. While in the previous experiments erosion and implantation processes were quantified by measuring the weight change of the sample, the new setup allows observing the evolution of the sample composition as a function of incident ion fluence. The present work discusses experimental results for $\mathrm{W}$ sputtering and $\mathrm{C}$ and $\mathrm{D}$ implantation as a function of the $\mathrm{C}$ fraction in the incident beams. The obtained experimental data are compared to simulations with the MonteCarlo code TRIDYN [11].

\section{Experimental}

The experiments were performed with mass-separated $12 \mathrm{keV} C_{2}^{-}$and $9 \mathrm{keV} D_{3}^{+}$ions with energies choosen to obtain maximum beam flux densities. It is assumed that each species projectile atom has an incidence energy of $6 \mathrm{keV}$ and $3 \mathrm{keV}$ respectively. The $\mathrm{C}-\mathrm{Kr}$ potential used in the TRIDYN code to describe the interaction between the projectiles/recoils and target atoms is a valid approximation for the choosen energies extending down to values of $\approx 50 \mathrm{eV}$, where the binary-collision approximation ultimately breaks down. Therefore, validation of the TRIDYN model at these energies allows finally to draw conclusions also for the lower ion energy range expected in fusion devices. Ion beam analysis (IBA) with $2.5 \mathrm{MeV}{ }^{3} \mathrm{He}$ ions was performed in-situ between the bombardment sessions, allowing the acquisition of the evolution of surface composition with increasing fluence. The use of $\mathrm{W}$ layers as a 
bombarded sample allows to use Rutherford back-scattering (RBS) for the measurement of the $\mathrm{W}$ areal density with an accuracy of $1 \%$. The change of $\mathrm{W}$ areal density is calculated as a difference between the initial and the post-bombardment values. The main error source is the uncertainty of the stopping power, which cancels out since the decrease of the areal density is measured relatively to the initial value. The amount of implanted $\mathrm{C}$ and $\mathrm{D}$ was measured using the nuclear reaction ${ }^{12} \mathrm{C}\left({ }^{3} \mathrm{He}, \mathrm{p}\right){ }^{14} \mathrm{~N}$ [12] and $\mathrm{D}\left({ }^{3} \mathrm{He}, \mathrm{p}\right) \alpha$ respectively. These measurements were evaluated using a reference a-C:D layer with known $\mathrm{D}$ and $\mathrm{C}$ areal densities with a resulting accuracy $<10 \%$. Further details of the experimental setup, as well as of the measurement technique are comprehensively described in [8].

The sample $\mathrm{W}$ layers (thickness $\approx 280 \mathrm{~nm} \pm 10 \%$ ) were deposited by magnetron onto $\mathrm{Si}$ single crystal wafers and onto polished $\mathrm{C}$ surfaces with an intermediate $\mathrm{Cu}$ layer (thickness $\approx 380 \mathrm{~nm} \pm 10 \%$ ) in the same process. The thickness of the $\mathrm{W}$ layer was always chosen to be large enough to prevent interaction of the projectiles with the other layers. The projectile ranges of $6 \mathrm{keV} \mathrm{C}$ and $3 \mathrm{keV} D$ are $R_{C}=10-15 \mathrm{~nm}$ and $R_{D}=40-50 \mathrm{~nm}$ respectively, depending on surface composition. Further details on structure and properties of the W layers can be found in [13]. Figure 1 shows images of the surface of W layer deposited on $\mathrm{C}$ and $\mathrm{Si}$ substrate respectively, obtained by atomic force microscopy. The $\mathrm{W}$ layer on $\mathrm{C}$ substrate sample shows much higher roughness level with RMS (root mean square) roughness of $\mathrm{R}_{\mathrm{a}}=32 \mathrm{~nm}$. Since $\mathrm{R}_{\mathrm{a}} \approx \mathrm{R}_{\mathrm{C}}, \mathrm{R}_{\mathrm{D}}$, the surface roughness must be taken into account. Samples with polished Si substrate are distinguished by much better smoothness with RMS roughness of $R_{a}=3.7 \mathrm{~nm}$. The surface of these samples can be assumed smooth, since $R_{a}<<R_{C}, R_{D}$ and surface effects can be neglected. The different surface roughness of the W layers is a direct result of the differences in the substrate material's surface roughness as the used Si single crystal wafers are smooth on an atomic scale while graphite was only mechanically polished. 


\section{Results and discussions}

\subsection{Bombardment with $C$ ions}

Figure 2 shows the change of the surface composition of both smooth (Si substrate) and rough surfaces (graphite substrate) in comparison to the initial state with increasing irradiation fluence. Open symbols denote the smooth surface, while filled symbols represent experimental data for the rough surface. The squares show the evolution of the concentration of implanted C. At the same time, W concentration decreases by sputtering. In comparison to the smooth $\mathrm{W}$ surface on $\mathrm{Si}$ substrate, the W layer on the C substrate shows a higher W sputter yield, as well as a slower C implantation rate, while $\mathrm{W}$ is still sputtered at a fluence of $2.2 \times 10^{22} \mathrm{~m}^{-2}$. In contrast, the smooth surface sample shows a coverage with a protective $\mathrm{C}$ layer at fluences $>7 \times 10^{21} \mathrm{~m}^{-2}$. The corresponding suppression of $\mathrm{W}$ sputtering is indicated by the constant $\mathrm{W}$ areal density.

Simulation with TRIDYN [11] shows that the evolution of the surface composition can be accurately reproduced for the smooth surface (on Si substrate), while experimental values for the rough surface (on graphite substrate) show a strong deviation. This behavior can be explained only by the presence of roughness, since other surface properties and bombardment parameters are identical. The approach suggested by M. Kuestner et.al. attributes a certain distribution of local incidence angles to each surface [14], [15]. However, in the present implementation of TRIDYN, this model cannot take into account the modification of surface composition and change of surface topography. The implementation of the effect of roughness in the current Monte-Carlo model will require a considerable research effort. Therefore, in a first attempt to understand this problem, the real angle distribution was approximated assuming an average incidence angle, which is not necessarily the same as the actual macroscopic value. The assumed angle can be interpreted as the most probable incidence angle in the angle distribution function. In our simulation shown in Figure 2 an 
incidence angle of $\alpha=38^{\circ}$ had to be used to obtain the best fit to the experimental data.

\subsection{Simultaneous bombardment with $D$ and $C$ ions}

The results of the bombardment of rough $\mathrm{W}$ surfaces with $\mathrm{D}$ and $\mathrm{C}$ ions at beam incidence angles of $\alpha=15^{\circ}$ are shown in Figure 3. The surface of the W layer under D bombardment is always rough because of layer blistering [13]. Figure 3(a) and (b) show the dependence of the W sputter yield $Y_{W}$ and of the equilibrium amount of $\mathrm{C}$ implanted, $C_{i m p l}$, as a function of $\mathrm{C}$ fraction, $f_{C}$, in the incident flux. Filled circles correspond to values measured after reaching equilibrium conditions, e.g. after the elemental composition does not change any more. Since after $f_{C}>13 \%$, surface is covered by protective C layer (infinite amount of implanted $\mathrm{C}$ at equilibrium), no $\mathrm{W}$ atoms can be sputtered any more, indicating the transition point from $\mathrm{W}$ sputtering to $\mathrm{C}$ layer growth. However, at $f_{C}=15 \%$ continuous $\mathrm{W}$ sputtering was also observed, which can result from deviations in surface roughness conditions. Thus, the transition point is located to be between $13 \%$ and $16.5 \%$ at the given experimental conditions. Open circles represent the initial value of $Y_{W}$ (Figure 3a), which correspond to extremely small fluences and can be obtained by extrapolation. At these conditions, a certain $\mathrm{C}$ amount is already implanted and the obtained $Y_{W}$ values can be used for the understanding of $\mathrm{W}$ sputtering by multi-species bombardment. The experimental data clearly indicate that both steady-state values of $Y_{W}$ and $C_{i m p l}$ increase with increasing $f_{C}$, until the transition point is reached.

TRIDYN simulation with an actual incidence angle of $\alpha=15^{\circ}$ results in a strong underestimation of $Y_{W}$ and an overestimation of $C_{i m p l}$. It also underestimates the transition point by a factor of 2 . This deviation indicates significant contribution of those effects, which are presently ignored by the model. Among the relevant process, only chemical interactions and/or the effect of roughness may lead to the observed deviations. In order to approximate 
the roughness effect, TRIDYN simulations with $\alpha=38^{\circ}$, taken from preliminary pure $\mathrm{C}$ bombardment experiment (see subsection 3.1), were performed. Although, the simulation cannot reproduce correctly the transition point, which is in the model found at $f_{C}>20 \%$, it approximates reasonably the experimental values of of $Y_{W}$ and $C_{i m p l}$. From this observations it can be concluded that the contribution of roughness is significant and should be studied more accurately, while the chemical interactions will play only a minor role in case of continuous W sputtering. One should take into account that chemical sputtering of implanted C by D shifts the transition point towards higher $f_{C}$ values, because of extra removal of $C$ from grown C layer.

The different masses of projectiles and $\mathrm{W}$ target atoms strongly reduce the energy transferred to the $\mathrm{W}$ atoms. W atoms may escape from the material only, if they gain sufficient momentum towards the surface and are close enough to the surface $(<10 \mathrm{~nm})$, to reach it before being completely stopped. Therefore, the bulk composition, as well as collisional cascades in the depth of the material can be neglected and $Y_{W}$ can be well approximated by the linear contribution of the $\mathrm{W}$ sputter yields due to $\mathrm{C}$ projectiles $Y^{C}=0.417$ (taken from the pure $\mathrm{C}$ bombardment experiment) and D projectiles $Y^{D}=0.0098$ [13]:

$$
Y_{W}=f_{C} \times Y^{C}+f_{D} \times Y^{D}
$$

where $f_{C}$ and $f_{D}$ are the $\mathrm{C}$ and $\mathrm{D}$ fractions in the incident flux. This approximation is further justified by the similar surface binding energies of $\mathrm{C}$ and W. However, the model cannot describe the target evolution from pure tungsten to the steady state mixed C/W surface and also cannot predict the transition point. It can only predict the value of $Y_{W}$ for $\mathrm{W}$ sputtering at steady state conditions. For C-fractions beyond the transition point to continous C-layer growth, the model is only valid for the initial pure $\mathrm{W}$ surface as long as the amount of implanted carbon is negligible. Furthermore the model breaks down at projectile energies 
where also $\mathrm{W}$ atoms from recoil cascades in the bulk have sufficient energy to escape the target.

Figure 3(c) shows the $\mathrm{D}$ retention in the $\mathrm{W}$ layer as a function of the $\mathrm{C}$ fraction in the total incident flux $(\mathrm{D}+\mathrm{C})$ after a total fluence of $4 \times 10^{22} \mathrm{~m}^{-2} \pm 30 \%$. Apart from $f_{C}=18 \%$, the $\mathrm{D}$ retention is approximately equal for all $f_{C}$ values. The scattering of the data is attributed to different surface roughness conditions, which may slightly vary between the samples. This similarity in D retention can be explained, taking into account the limited depth of the W layer, and the presence of $\mathrm{Cu}$ as a diffusion barrier. These factors lead to limited number of traps, depending only on the thickness of the $\mathrm{W}$ layer (since structure of all layers is the same). Since D atoms cannot diffuse into the substrate, all D is trapped within the depth range of the analysing technique. In addition to voids and displacement traps in $\mathrm{W}$, the fast growth of the C layer provides chemical bonding of D in grown C-D films, which increases strongly the amount of $\mathrm{D}$ retained at $f_{C}=18 \%$.

\section{Conclusions}

Sputtering of $\mathrm{W}$ and implantation of $\mathrm{C}$ and $\mathrm{D}$ were studied as a function of the $\mathrm{C}$ fraction in the incident flux of $\mathrm{C}$ and $\mathrm{D}$ ions. Preliminary experiments with pure $\mathrm{C}$ bombardment show that surface roughness can increase the sputter yield of $\mathrm{W}$ and decrease the $\mathrm{C}$ implantation rate. With respect to the latter, the roughness produces a similar effect than chemical sputtering resulting in elevated sputtering of implanted $\mathrm{C}$, so that these processes cannot be easily separated [9]. Increased sputtering of $C$ is the main reason of the shift of the transition point from $\approx 7 \%$ predicted for smooth surfaces to $\approx 15 \%$ for experimental rough surfaces, because the enhanced erosion at the resulting higher incidence angles inhibits the growth of a protective C layer. TRIDYN simulations provide a reasonable approximation of the experimental results with the assumption of an effective mean incidence angle, which 
indicates that the roughness effect is the most significant contribution to the observed phenomena. At the same time, chemical erosion of implanted $\mathrm{C}$ plays only a minor role at steady-state $\mathrm{W}$ erosion. For steady state conditions, the sputtering of $\mathrm{W}$ surfaces by incident $\mathrm{D}+\mathrm{C}$ can also be approximated by the linear contribution of the incident species to the total sputter yield. At the same time D retention is limited by the number of available traps, so no significant differences in retention can be observed in experiments with $\mathrm{W}$ layers.

\section{Acknowledgements}

The authors would like to thank Drs. J. Roth, V. Alimov, and M. Mayer for their suggestions and helpful discussions; Dr. A . Gigler for AFM measurements; P. Matern, G. Matern, S. Lindig, M. Fusseder, J. Dorner for valuable technical assistance. 


\section{References}

[1] ITER Physics Base, Nucl. Fus. 39 (1999) 2137-2638

[2] G. Janeschitz, ITER JCT and ITER HTs, J. Nucl. Mat. 290-293 (2001) 1

[3] D. Meade, et al., Mission and design of the fusion ignition research experiment, in: Proceedings of the 18th IAEA Conf. on Fusion Energy, Sorrento, Italy October, 2000, (CD-ROM), pp. IAEA-CN-77/FTP2/16, IAEA, Vienna, 2001.

[4] S. Nishio, et al., Conceptional design of advanced steady-state tokamak reactor, in: Proceedings of the 18th Conf. on Fusion Energy, Sorrento, Italy October, 2000, (CDROM), pp. IAEA-CN-77/FTP2/14, IAEA, Vienna, 2001.

[5] K. Krieger et al., J. Nucl. Mat. 266 -269 (1999) 207

[6] J.P. Biersack, Radiation Effects, 19 (1973) 249

[7] K.Krieger, J.Roth, J. of Nucl. Mat., 290-293 (2001) 107-111

[8] I. Bizyukov, K. Krieger. Rev. Sci. Instrum. 77 (2006) 043501

[9] K.Schmid, J.Roth, J. of Nucl. Mat. 313 -316 (2003) $302-310$

[10] A.A. Haasz, J. Davis, Nucl. Instrum. and Methods in Phys. Res. B83. (1993) 117.

[11] W. Moeller, W. Eckstein, J.P. Biersack, Comput. Phys. Commun. .51 (1988) 355.

[12] S.Y. Tong, W.N. Lennard, P.F.A. Alkemada, I.V. Mitchell, Nucl. Instrum. Methods B45 (1990) 41

[13] I. Bizyukov, K. Krieger, N. Azarenkov, S. Levchuk, Ch. Linsmeier, J. Nucl. Mat. 337$339(2005) 965$. 
[14] M. Küstner, W. Eckstein, V. Dose, J. Roth. Nucl. Instrum. and Methods in Phys. Res., B145 (1998) 320

[15] M. Küstner, W. Eckstein, E. Hechtl, J. Roth. J. Nucl. Mat. 265 (1999) 22-27 


\section{List of figure captions}

Figure 1. Surface topography taken by AFM of (a) rough surface (W on C) and (b) smooth surface (W on Si). Axis units are microns. View angle is parallel to the horizontal plane of the surface.

Figure 2. Change of surface layer composition under bombardment with $\mathrm{C}$ ions at normal incidence angle. The graph shows the areal densities of $\mathrm{C}$ and $\mathrm{W}$ as a function of incident C fluence. Points denote experimental data, while solid and dotted curves represent TRIDYN calculations for normal incidence angle and $38^{\circ}$ incidence angle respectively.

Figure 3. Sputter yield of tungsten, $Y_{W}$, (a), amount of implanted carbon, $C_{i m p l}$, (b) and amount of retained D (c) as a function of the C fraction in the total incident flux of D and C ions. The results were obtained using samples with W layers on graphite substrate. 


\section{List of figures}

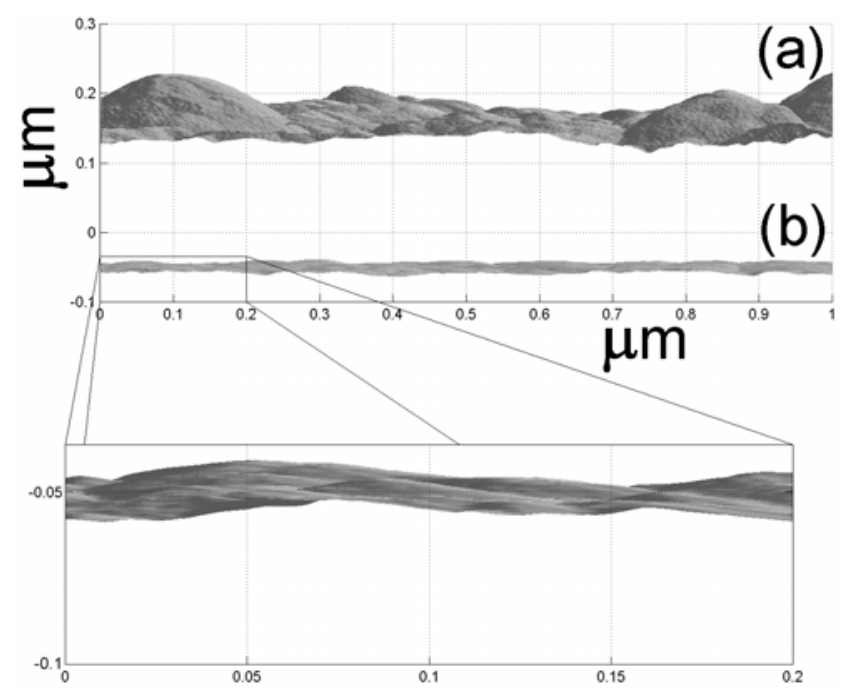

Figure 1 


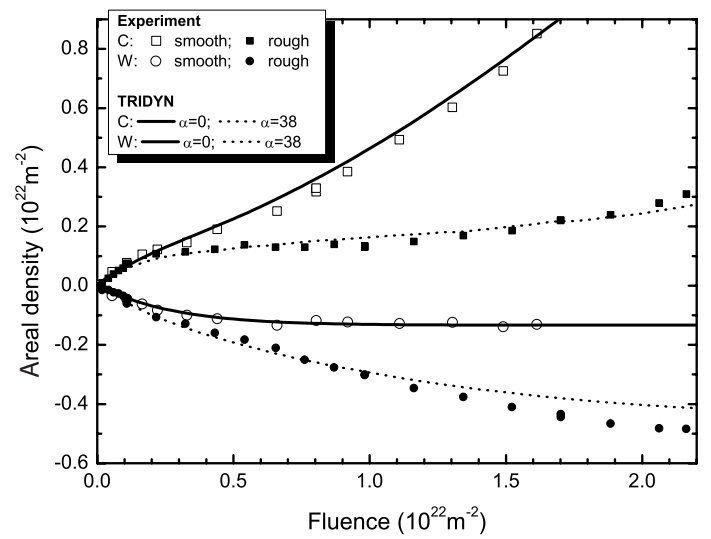

Figure 2

Page 15 


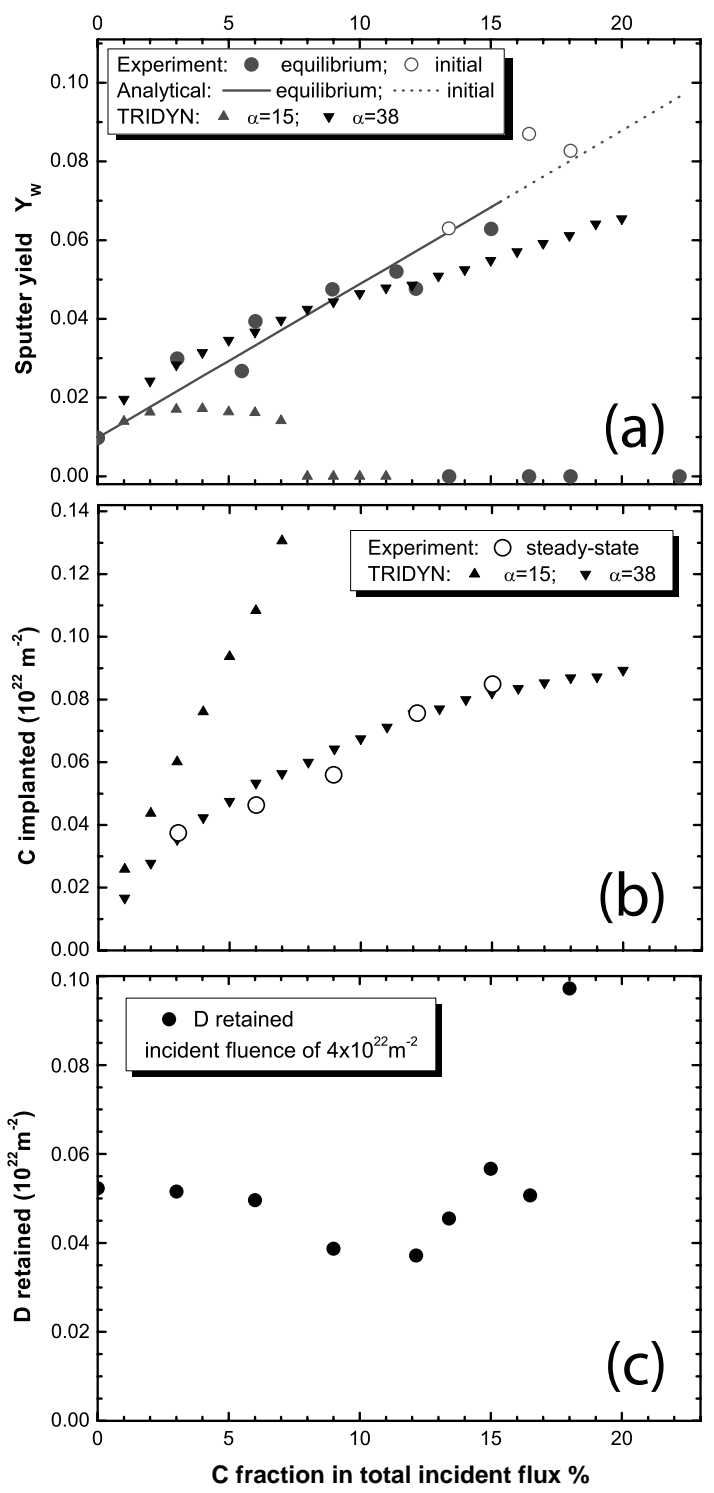

Figure 3 